\title{
EDITORIAL
}

\section{ARTICLES PUBLISHED IN THE JANUARY/FEBRUARY 2011 ISSUE OF THE "REVISTA DO INSTITUTO DE MEDICINA TROPICAL DE SÃO PAULO”}

In the 2011 January/February issue of the Revista do Instituto de Medicina Tropical de São Paulo (Journal of the São Paulo Institute of Tropical Medicine) RODRIGUES et al. studied a group of azole antimycotics, clotrimazole, ketoconazole and fluconazole for their abilities to inhibit beta-hematin synthesis and hemoglobin proteolysis in vitro in a context of hemoglobin degradation by Plasmodium parasites. Compounds were also assayed in vivo in a malaria murine model. Clotrimazole and ketoconazole inhibited both hematin synthesis and hemoglobin proteolysis. The results indicated that the inhibition of the hemoglobin metabolism by the azole antimycotics could be responsible for their antimalarial effect.

SCURACCHIO et al. described a transfusion-transmitted malaria case in São Paulo, Brazil, where one recipient received infected blood and developed the disease. Thick blood smears confirmed Plasmodium malariae. All donors lived in S. Paulo and one of them showed positive hemoscopy and PCR DNA assay revealed P. malariae in the donor's blood. Furthermore donor serum was IFAT positive and the $P$. vivax ELISA was reactive. This case suggests the need of reviewing donor selection criteria and deferral strategies to prevent possible cases of transfusion-transmitted malaria.

DRUMOND \& COSTA reviewed the situation of visceral leishmaniasis in Teresina, Piauí State, during the last 40 years. The typically rural disease began to appear in urban areas over time. Teresina, capital of Piauí State, was affected by the first epidemic of visceral leishmaniasis in Brazil. Human population growth and migration to urban areas contributed to vector proliferation. At present the incidence of human and canine disease is quite high and uncontrolled. Despite previously conducted studies, little is known about visceral leishmaniasis epidemiology in urban areas.

ALMEIDA et al. isolated rabies virus from a colony of Eptesicus furinalis bats adapted to live in urban buildings. Three dead bats were found in the yard of a house in an urban area of Jundiai city, S. Paulo State, Brazil and two of the three bats tested positive for rabies. A large colony of Eptesicus furinalis was found in the house attic and of the 119 bats captured, four more tested positive for rabies.

BRITO et al. report on an antemortem and postmortem diagnosis of rabies in a veterinarian who became infected when handling hervibores with rabies. The antemortem diagnosis was carried out with a saliva sample and a biopsy of hair follicles which was found to be positive by RT-PCR. Genetic sequencing showed that the infection was caused by variant 3 (Desmodus rotundus) a finding confirmed by tests on the brain sample obtained postmortem.
LIVRAMENTO et al. tried to determine the seroprevalence of HBV and HCV markers among children and adolescents and verify the epidemiology of the HBV infection over than a decade of the introduction of vaccination program. 393 samples were obtained and seropositivity for $\mathrm{HBsAg}$ was $0.76 \%$ and for total anti-HBc was $1.02 \%$. Copositivity between HBsAg and total anti-HBc was verified in $0.76 \%$ of the analyzed samples. There was no seropositivity for anti-HCV marker. The seroprevalence of HBV infection markers among children and adolescents in Southern Brazil is high compared to that reported in other countries.

FRAGA et al. analyzed the genetic polymorphism of Triatoma flavida and Triatoma bruneri, which are Cuban species of Triatominae (Hemiptera: Reduviidae), using RAPD techniques. The genetic flow among them was calculated. The dendogram based on calculated Jaccard distances showed two clearly distinguishable clusters which coincided with the studied species. Within each species, moderate genetic differentiation (Fst 0.05-0.15) and migration rates $(n>1)$ were found among populations that reveal gene flow and genetic homogeneity. Between species the Fst value showed a high genetic differentiation and the migration rate was insufficient to maintain genetic homogeneity and confirmed the absence of gene flow between them. Results confirm genetic variability among the studied species.

CARNEIRO et al. investigated the susceptibility of Cebus apella monkeys to experimental Leishmania infantum chagasi infections, the etiologic agent of American visceral leishmaniasis. Monkeys were inoculated either by intra-dermal via with promastigotes forms from phase culture medium or with amastigotes from viscera of infected hamsters. Intravenous and intra-peritoneal via were used. Parameters of infection were physical examination, needle aspiration of blood marrow, indirect fluorescence antibody test and delayed type hypersensitivity. Results were, as a whole, irregular and the authors do not encourage the use of Cebus apella monkeys as animal model for studying American visceral leishmaniasis.

MENTZ et al. reported the first case of human ocular sparganosis in the state of Santa Catarina, southern Brazil. Patient presented three moveable inflammatory masses in her right eye during two years. By surgical excisional biopsy a helminth larval stage was removed and identified as sparganum.
Thales de Brito, Editor Pedro Paulo Chieffi, Associate Editor Marcello F. Franco, Associate Editor 